\title{
Spherical Combustion Clouds in Explosions
}

\author{
Allen L. Kuhl ${ }^{1, *}$, John B. Bell ${ }^{2}$, Vincent E. Beckner ${ }^{2}$ \& Kaushik Balakrishnan ${ }^{2}$ \\ ${ }^{1}$ Lawrence Livermore National Laboratory \\ 7000 East Avenue, Livermore, California, USA 94551 \\ ${ }^{2}$ Lawrence Berkeley National Laboratory \\ 1 Cyclotron Road, Berkeley, California, USA 94551
}

\section{Introduction}

This study investigates combustion clouds embedded in unconfined spherical explosions. Two charge configurations are considered: a $0.5-\mathrm{g}$ spherical PETN booster surrounded by a spherical shell of either 1-g of TNT solid or of 1-g of Aluminum (Al) powder; these provide the fuel for the combustion process. Detonation of booster disperses the fuel, whose expansion drives a blast wave into the surrounding atmosphere. The fuel-air interface is unstable and rapidly evolves into a turbulent mixing layer. The hot detonation products and the shock-heated air serve as ultra-strong ignition sources of the fuel-air mixture, which evolves into a spherical combustion cloud $[1,2]$. The evolution of the blast wave and ensuing combustion cloud dynamics are studied via numerical simulations with our two-phase Adaptive Mesh Refinement (AMR) combustion code [3,4].

\section{Model}

\section{Conservation Laws}

The Model is based on the Eulerian multi-phase conservation laws for a dilute heterogeneous continuum, as formulated by Nigmatulin [6]. We model the evolution of the gas phase combustion fields in the limit of large Reynolds and Peclet numbers, where effects of molecular diffusion and heat conduction are negligible. The flow field is governed by the gas-dynamic conservation laws:

Mass:

$$
\partial_{t} \rho+\nabla \cdot(\rho \mathbf{u})=\dot{\sigma}_{s}
$$

Momentum:

$$
\partial_{t} \rho \mathbf{u}+\nabla \cdot(\rho \mathbf{u u}+p)=\dot{\sigma}_{s} \mathbf{v}-\dot{f}_{s}
$$

Energy:

$$
\partial_{t} \rho E+\nabla \cdot(\rho \mathbf{u} E+p \mathbf{u})=-\dot{q}_{s}+\dot{\sigma}_{s} E_{s}-\dot{f}_{s} \cdot \mathbf{v}
$$

Where $\rho, p, U$ represent the gas density, pressure and specific internal energy, $\mathbf{u}$ is the gas velocity vector, and $E \equiv U+\mathbf{u} \cdot \mathbf{u} / 2$ denotes the total energy of the gas phase. Source terms on the right hand side take into account: mass addition to gas phase due to particle burning $\left(\dot{\sigma}_{s}\right)$, particle $\operatorname{drag}\left(\dot{f}_{s}\right)$, and heat losses $\left(\dot{q}_{s}\right)$.

We treat the particle phase as a Eulerian continuum field [6]. We consider the dilute limit, devoid of particle-particle interactions, so that the pressure and sound speed of the particle phase are zero. We model the evolution of particle phase mass, momentum and energy fields by the conservation laws of continuum mechanics for heterogeneous media: 
Mass:

$\partial_{t} \sigma+\nabla \cdot \sigma \mathbf{v}=-\dot{\sigma}_{s}$

Momentum:

$\partial_{t} \sigma \mathbf{v}+\nabla \cdot \sigma \mathbf{v} \mathbf{v}=-\dot{\sigma}_{s} \mathbf{v}+\dot{f}_{s}$

Energy:

$$
\partial_{t} \sigma E_{s}+\nabla \cdot \sigma E_{s} \mathbf{v}=\dot{q}_{s}-\dot{\sigma}_{s} E_{s}+\dot{f}_{s} \cdot \mathbf{v}
$$

where $\sigma$ and $\mathbf{v}$ represent the particle-phase density and velocity, and $E_{s} \equiv C_{s} T_{s}+\mathbf{v} \cdot \mathbf{v} / 2$ denotes the total energy of the particle phase.

\section{Interactions}

The inter-phase interaction terms for mass, momentum, heat and particle burning law take the form as described by Veyssiere and Khasainov [7]:

$$
\begin{array}{ll}
\text { Mass Exchange: } & \dot{\sigma}_{s}=\left\{\begin{array}{ll}
0 & T_{s}<T_{i g n} \\
-3 \sigma\left(1+0.276 \sqrt{\operatorname{Re}_{s}}\right) / t_{s} & T_{s} \geq T_{i g n}
\end{array}:\right. \\
\text { Momentum Exchange: } & \dot{f}_{s}=\left(3 \rho \sigma / 4 \rho_{s} d_{s}\right) C_{D}(\mathbf{u}-\mathbf{v})|\mathbf{u}-\mathbf{v}| \\
\text { Drag Coefficient: } & C_{D}=24 / \operatorname{Re}_{s}+4.4 / \sqrt{\operatorname{Re}_{s}}+0.42 \text { and } \operatorname{Re}_{s}=\rho d_{s}|\mathbf{u}-\mathbf{v}| / \mu \\
\text { Heat Exchange: } & \dot{q}_{s}=\left(6 \sigma / \rho_{s} d_{s}\right)\left[N u \lambda\left(T-T_{s}\right) / d_{s}+\varepsilon \sigma_{\text {Boltz }}\left(T^{4}-T_{s}^{4}\right)\right] \\
\text { Convective Heat Transfer: } & N u=2+0.6 \operatorname{Pr} \sqrt{\operatorname{Re}_{s}} \\
\text { Ingignoli Burning Law (1999): } & t_{s}=K d_{s 0}^{n} / \phi^{0.9}
\end{array}
$$

\section{Combustion}

We consider three fuels: PETN detonation products $\left(F_{1}\right)$, TNT detonation products $\left(F_{2}\right)$ and Aluminum $\left(F_{3}\right)$, and their corresponding combustion products: PETN-air $\left(P_{1}\right)$, TNT-air $\left(P_{2}\right)$, and Alair $\left(P_{3}\right)$. We model the combustion of fuel $F_{k}$ with air $(A)$ yielding equilibrium products $P_{k}$ :

$$
F_{k}+A \Rightarrow P_{k} \quad(k=1,2 \text { or } 3)
$$

The mass fractions $Y_{k}$ of the components are governed by the following conservation laws:

Fuel-k:

$$
\begin{array}{ll}
\text { Fuel-k: } & \partial_{t} \rho Y_{F k}+\nabla \cdot \rho Y_{F k} \mathbf{u}=-\dot{s}_{k}+\delta_{k 2} \dot{\sigma}_{k} \\
\text { Air: } & \partial_{t} \rho Y_{A}+\nabla \cdot \rho Y_{A} \mathbf{u}=-\sum_{k} \alpha_{k} \dot{s}_{k} \\
\text { Products-k: } & \partial_{t} \rho Y_{P k}+\nabla \cdot \rho Y_{P k} \mathbf{u}=\sum_{k}\left(1+\alpha_{k}\right) \dot{s}_{k}
\end{array}
$$

Fuel and air are consumed in stoichiometric proportions: $\alpha_{k}=A / F_{k}$. In the above, $\dot{s}_{k}$ represents the global kinetics sink term. In this work we use the fast-chemistry limit that is consistent with the inviscid gas-dynamic model (1)-(3), so whenever fuel and air enter a computational cell, they are consumed in one time step. Here $\delta_{k 2}$ represents the Kronecker delta $\left(\delta_{k 2}=0\right.$ if $k=1$ and $\delta_{k 2}=1$ if $k=2$ ) and takes into account the vaporization of $\mathrm{Al}$ fuel from the particle phase $\mathrm{EQ}$. (4), which creates a source of Al fuel in the gas phase.

\section{Equations of State}

The thermodynamic states encountered during SDF explosions have been analyzed in by Kuhl and Khasainov [8]. The locus of states of component $c$ in specific internal energy-temperature plane are fit with quadratic functions of temperature:

$$
U_{c}=a_{c} T^{2}+b_{c} T+c_{c}
$$

For cells containing a mixture of components, the mixture energy also satisfies a quadratic form:

$$
U_{m}(T)=\sum_{c} Y_{c} U_{c}=a_{m} T^{2}+b_{m} T+c_{m}
$$

Given the mixture specific internal energy $U_{m}$ the mixture temperature can be evaluated by: 


$$
T_{m}=\left[-b_{m}+\sqrt{b_{m}^{2}-4 a_{m}\left(c_{m}-U_{m}\right)}\right] / 2 a_{m}
$$

using mixture coefficients as defined by:

$$
a_{m}=\sum_{c} Y_{c} a_{c}, b_{m}=\sum_{c} Y_{c} b_{c}, c_{m}=\sum_{c} Y_{c} c_{c}, R_{m}=\sum_{c} Y_{c} R_{c}
$$

For pure cells, the pressure of a component is calculated from the perfect gas relation $p_{c}=\rho_{c} R_{c} T_{c}$, or from the JWL function in the detonation products gases [4]. In mixed cells, the pressure is calculated from the mixture temperature by the "law of additive pressures":

$$
p_{m}=\sum_{c} p_{c}\left(V_{m}, T_{m}\right)
$$

where $p_{c}\left(V_{m}, T_{m}\right)$ denotes the pressure of component $c$ if it existed alone at $V_{m}$ and $T_{m}$.

\section{Numerical Methods}

The governing equations (1)-(6) and (14)-(16) are integrated with high-resolution upwind methods that represent high-order generalizations of Godunov's method. The algorithm for gas phase conservation laws is based on an efficient Riemann solver for gas-dynamics first developed by Colella and Glaz [9]. The algorithm for the particle phase conservation laws is based on a Riemann solver for two-phase flows as developed by Collins et al. [10]. Source terms are treated with operator splitting methods. Being based on Riemann solvers, information propagates along characteristics at the correct wave speeds, and they incorporate nonlinear wave interactions within the cell during the time step.

These Godunov schemes have been incorporated into an adaptive mesh refinement (AMR) algorithm of Berger \& Colella [11] that allows us to focus computational effort in complex regions of the flow such as mixing layers and reaction zones. In this AMR approach, regions to be refined are organized into rectangular patches, with 100's to 1,000's of grid-points per patch. AMR is also used to refine turbulent mixing regions; by successive refinements we are able to capture the energy-bearing scales of the turbulence on the computational grid. In this way we are able to compute the effects of turbulent mixing without resorting to turbulence modeling (which is not applicable to this problem). This is consistent with the "MILES" approach of Boris et al. [12].

\section{Results}

Turbulent combustion inside the cloud is visualized by a color-bar representation of the temperature field in Fig. 1. The TNT-air cloud reaches a combustion temperature of $\sim 2,000 \mathrm{~K}$, while the Al-air cloud reaches a combustion temperature between 3,000 and 4,000 K. For the TNT case, combustion occurs along thin flame sheets (Fig. 2), while a distributed-combustion mode is realized for the Al-powder case (Fig. 1 d-f) due to two-phase flow effects.

By performing reactive and non-reactive flow simulations shown in Fig. 3, it was demonstrated that combustion caused no change in the pressure field during the positive phase of the blast wave-in other words, combustion in the fireball is isobaric. This finding was confirmed by experimental pressure measurements [5].

In these unconfined cases shown in Fig. 4, fuel consumption was limited to $\sim 60 \%$, while for confined cases $[3,4]$ fuel consumption approached $100 \%$ due to continued mixing induced by shock reverberations in the chamber.

Taking advantage of the point symmetry inherent in this problem, the flow field was azimuthally averaged to extract the mean fields: $\bar{\Phi}(r, t)$ and their r.m.s. fluctuations: $\bar{\Phi}(r, t)_{r m s}$; ensemble size is shown in Table 1. These were used to construct the evolution of the mean and r.m.s. profiles, the Reynolds stress profiles, and the turbulent kinetic energy spectrum of the combustion clouds. Some of the mean and rms profiles are shown in Figs. 6 and 7; all will be included in the final manuscript ${ }^{1}$.

\footnotetext{
${ }^{1}$ Due to page limitations, all results cannot be included in this extended Abstract.
} 


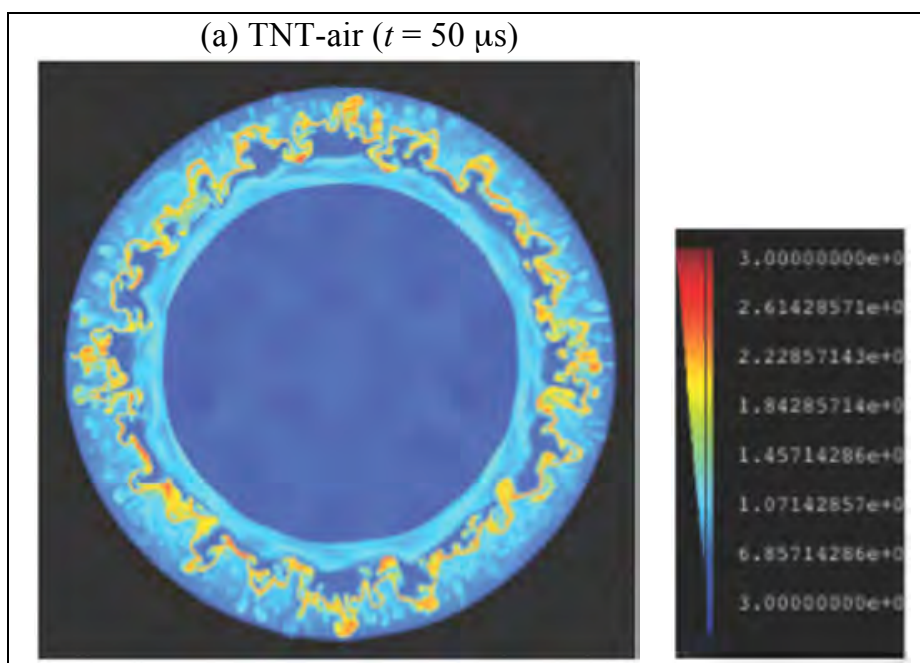

(b) TNT-air $(t=110 \mu \mathrm{s})$

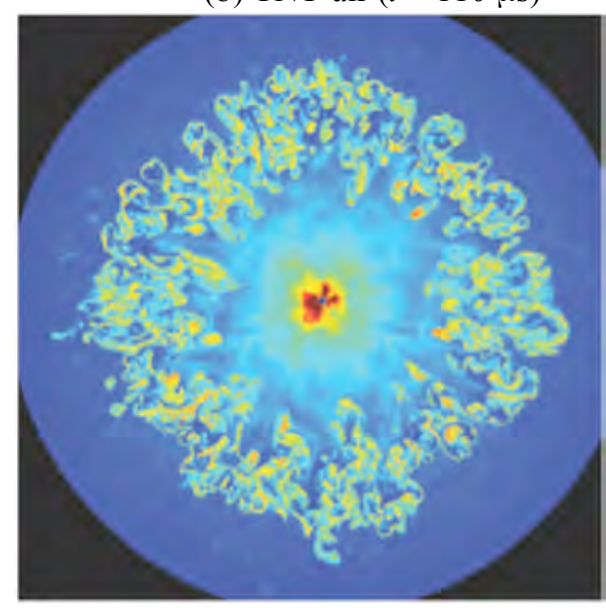

(c) TNT-air $(t=410 \mu \mathrm{s})$

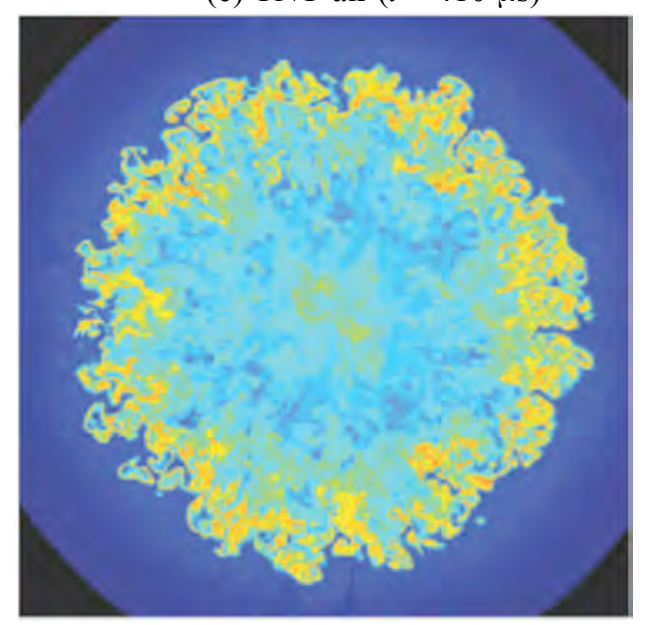

(d) Al-air $(t=50 \mu \mathrm{s})$

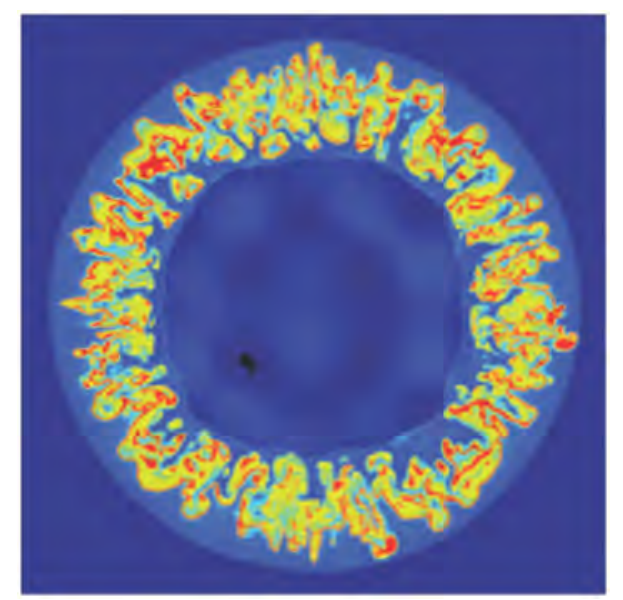

(e) Al-air $(t=110 \mu \mathrm{s})$

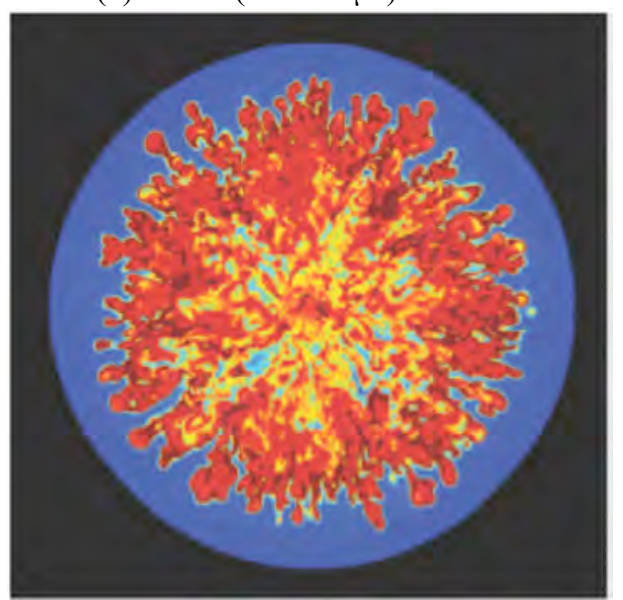

(f) Al-air $(t=410 \mu \mathrm{s})$

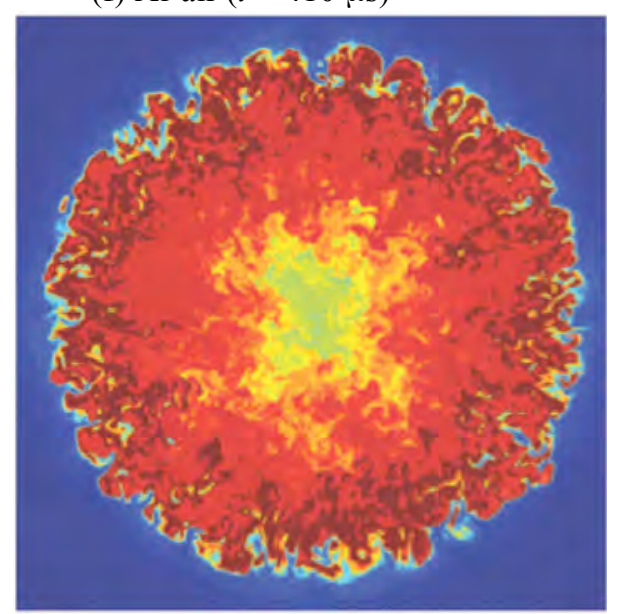

Figure 1. Cross-sectional view of the temperature field of spherical combustion clouds: frame $a-c$ illustrate TNT-air combustion while frames $d-f$ depict Al-air combustion (colors denote temperature - red: $3,000<T<4,000 \mathrm{~K}$; yellow: $T=2,000 \mathrm{~K}$; turquoise: $\mathrm{T}=1,000 \mathrm{~K}$ ). 


\section{Conclusions}

The characteristic temperature in the TNT-air combustion cloud is around 2,000 K, while the temperature in the Al-air combustion cloud is between $3,000 \mathrm{~K}$ and $4,000 \mathrm{~K}$. This trend is consistent with the adiabatic flame temperature of 2,900 K for stoichiometric TNT-air combustion and 4,120 K for stoichiometric Al-air combustion. Combustion occurs along thin flame sheets for the TNT case, while a distributed mode of combustion is observed for the Al-air case; the latter is attributed to a twophase (slip-flow) effect. In such unconfined explosions, combustion is isobaric, and thus has no effect of the blast wave pressure distribution during the positive phase. This finding has been confirmed by pressure measurements of such blast waves [5]. The fuel consumption was limited to $\sim 60 \%$ in these unconfined explosions. This corresponds to the amount of air the cloud can entrain by turbulent mixing in spherical explosions. This seems to be an inherent property of such spherical mixing layers. This contrasts with confined combustion in calorimeters, where fuel consumption was more that $90 \%$ for the same fuels $[1,2,3]$ as a result of the continued mixing induced by shock reverberations in the chamber.

\section{References}

[1] A. L. Kuhl, H. Reichenbach, Combustion effects in confined explosions, Proc. Combustion Institute 32 (2) 2009 pp. 2291-2298.

[2] A. L. Kuhl, H. Reichenbach, Barometric calorimeters, Comb., Exp. \& Shock Waves, 4 (2) 2010 pp. 271-278.

[3] A. L. Kuhl, J. B. Bell, V. E. Beckner, Heterogeneous continuum model of aluminum particle combustion in explosions, Combustion Explosion and Shock Waves 46 (4), pp. 433-448, 2010.

[4] A. L. Kuhl, J. B. Bell, V. E. Beckner, H. Reichenbach, Gasdynamic model of turbulent combustion in TNT explosions, Proc. Combustion Institute 33 (II) 2010, pp. 2177-2185.

[5] A. L. Kuhl, H. Reichenbach, J. B. Bell, V. E. Beckner, Reactive blast waves from composite charges, $14^{\text {th }}$ Int. Detonation Symposium, Office of Naval Research ONR-351-10185, 2011, pp 806-815.

[6] R. I. Nigmatulin Dynamics of multi-phase flows Vol. 1, Moscow, Nauka, 1987, 464 pp.

[7] B. Veyssiere, B. Khasainov, A model for steady, plane, double-front detonations (DFD) in gaseous explosive mixtures with Al particles in suspension, Combust. \& Flame, 85 (1,2), 1991, pp. 241-253.

[8] A. L. Kuhl \& B. Khasainov, Quadratic model of thermodynamic states in SDF explosions, Energetic Materials: $38^{\text {th }}$ Int. Conf. ICT, 2007, pp. 143.1-143.11.

[9] P. Colella \& H. Glaz, Efficient solutionaAlgorithms for the Riemann problem for real gases, J Comp. Phys., 59, 1985, pp. 264-289.

[10] P. Collins, R. E. Ferguson, K. Chien, A. L. Kuhl, J. Krispin \& H. M. Glaz, Simulation of shock-induced dusty gas flows using various models, AIAA 94-2309, 1994.

[11] M. J. Berger, P. Colella, Local adaptive mesh refinement for shock hydrodynamics, J. Comp. Phys. 82 (1), 1989, pp. 64-84.

[12] J. Boris et al., New insights into large eddy simulation, Fluid Dynamics Research 10, 1992, pp190-228.

(a) flow field

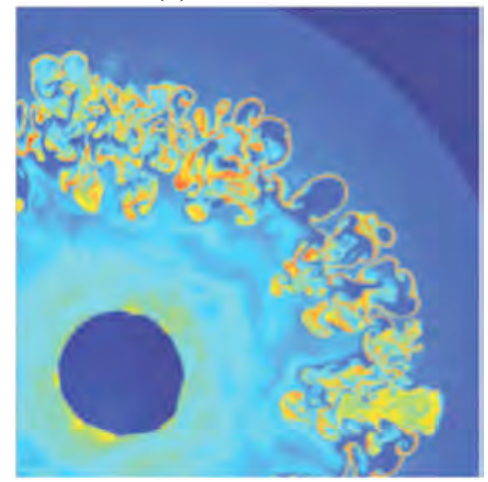

(b) blow-up near front

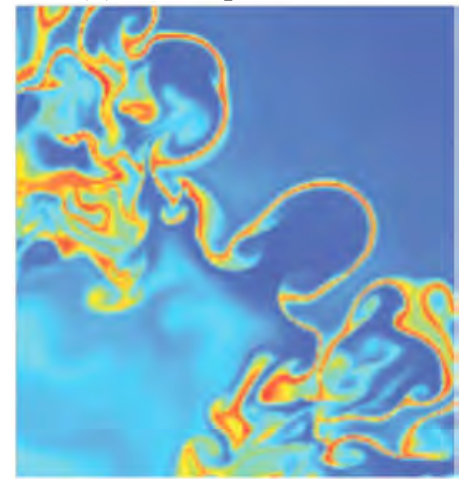

Table 1. Ensemble size, $N^{*}$

\begin{tabular}{|c|c|}
\hline$r(\mathrm{~cm})$ & $N=4 \pi(r / \Delta)^{2}$ \\
\hline 1 & 2,000 \\
5 & $0.5 \times 10^{5}$ \\
10 & $2 \times 10^{5}$ \\
15 & $4.6 \times 10^{5}$ \\
20 & $8 \times 10^{5}$ \\
\hline
\end{tabular}

* for $\Delta_{2}=0.8 \mathrm{~mm}$

Figure 2. Exothermic sheet in the TNT-air combustion field (100 $\mu$ s). 


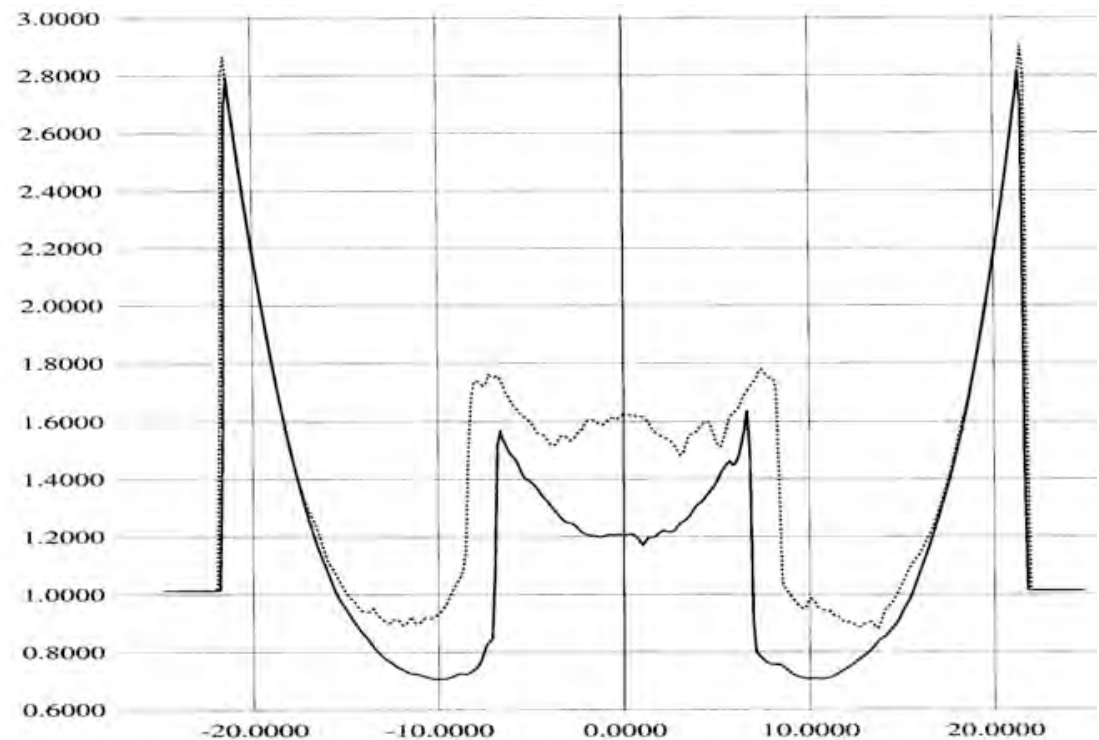

Figure 3. Blast wave pressure distribution $p(x, t=100 \mu s)$ along the $x$-axis at for a TNT explosion in air: dotted curve denotes combustion with air while solid curve represents the no combustion case.

(a) TNT-air combustion

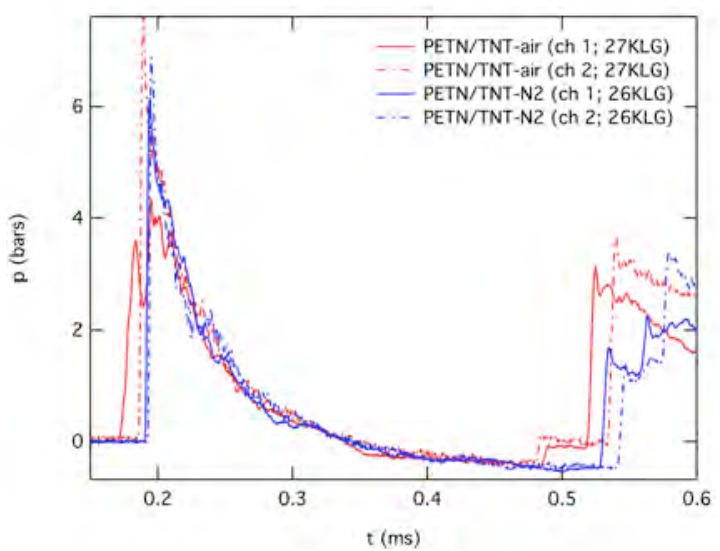

(b) Al-air combustion

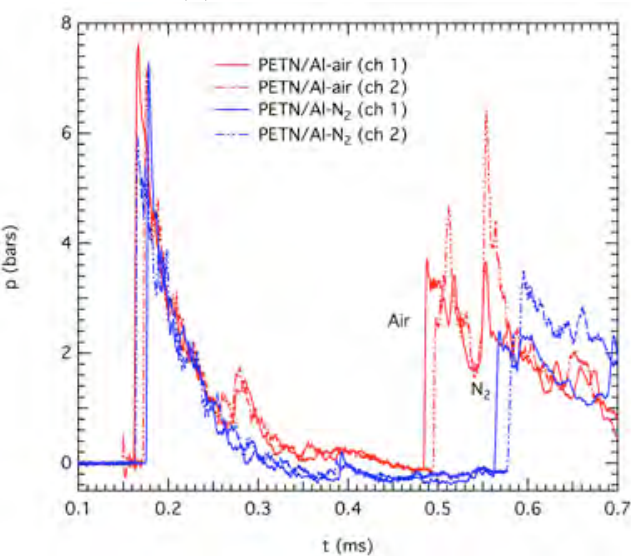

Figure 4. Blast wave pressure histories measured on the lid of calorimeter $\mathrm{C}$ for TNT and Al SDF charges; red curves represent combustion in air while blue curves represent the explosions in $\mathrm{N}_{2}$ [1].

(a) TNT-air combustion

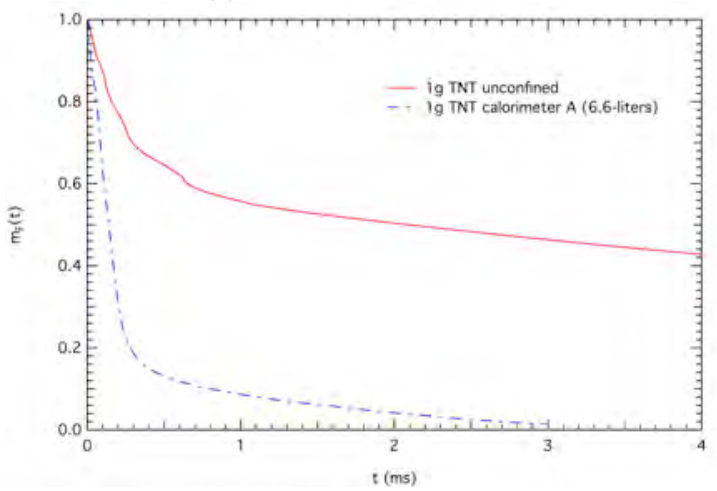

Figure 5. Fuel consumption over time: (a) TNT-air combustion; (b) Al-air combustion. (b) Al-air combustion

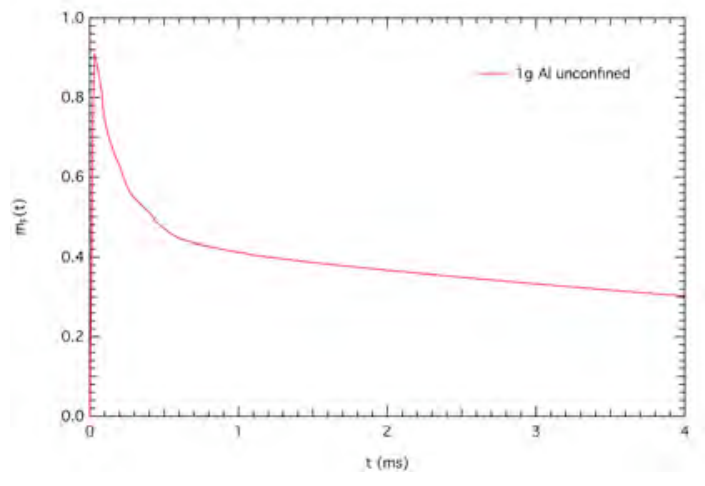




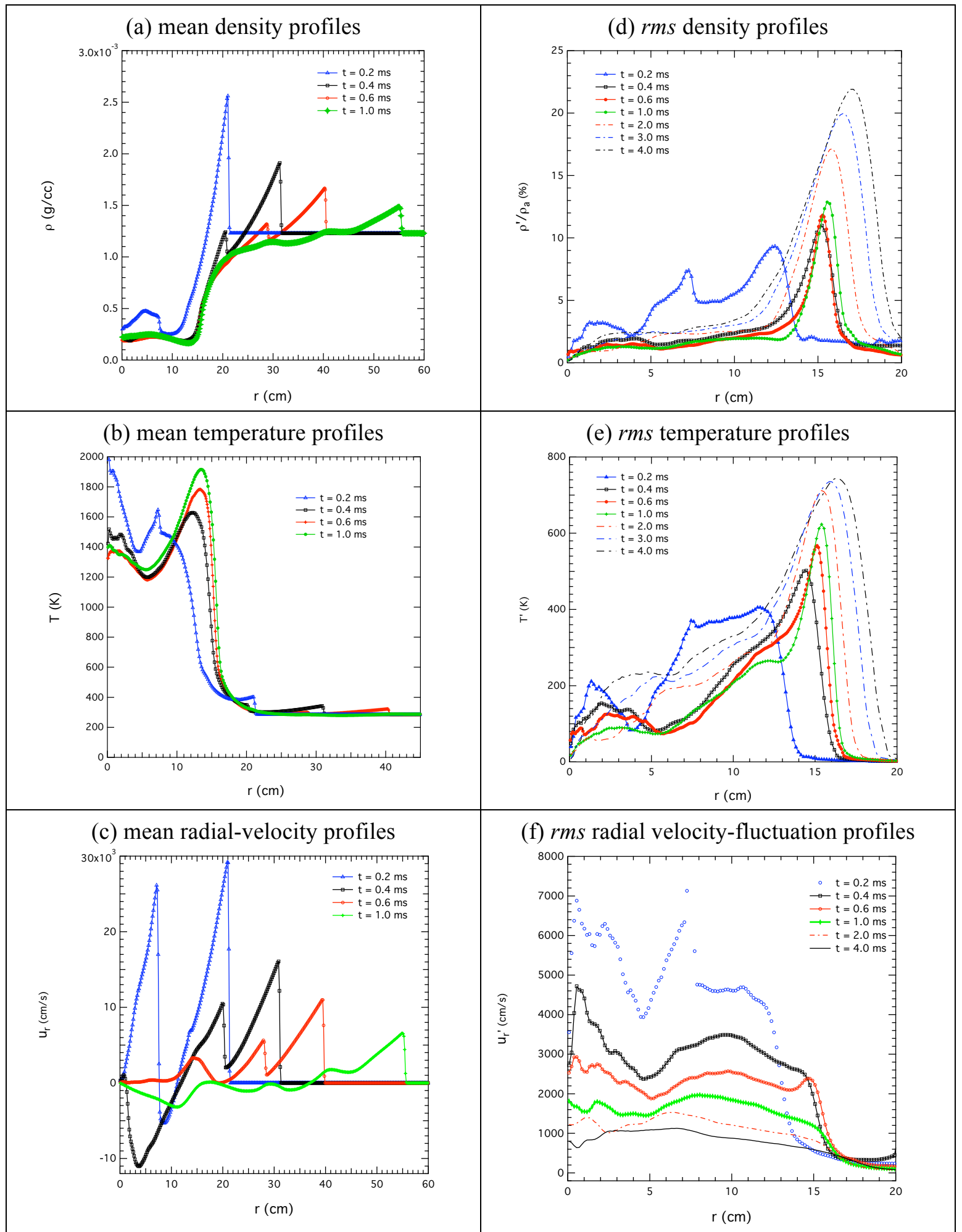

Figure 6. Evolution of the mean and rms profiles in the TNT combustion cloud (continued). 


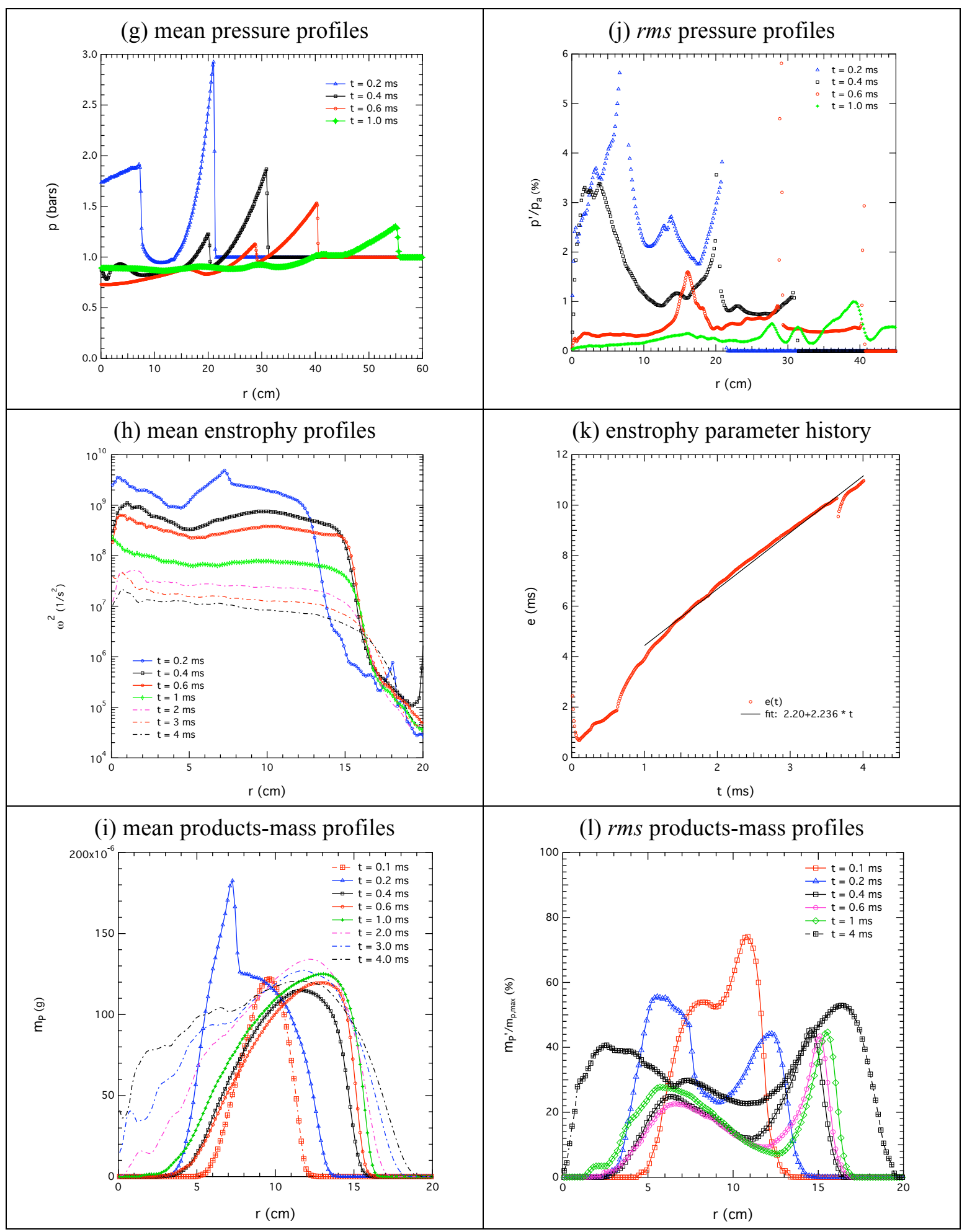

Figure 6. Evolution of the mean and $r m s$ profiles in the TNT combustion cloud (concluded). 


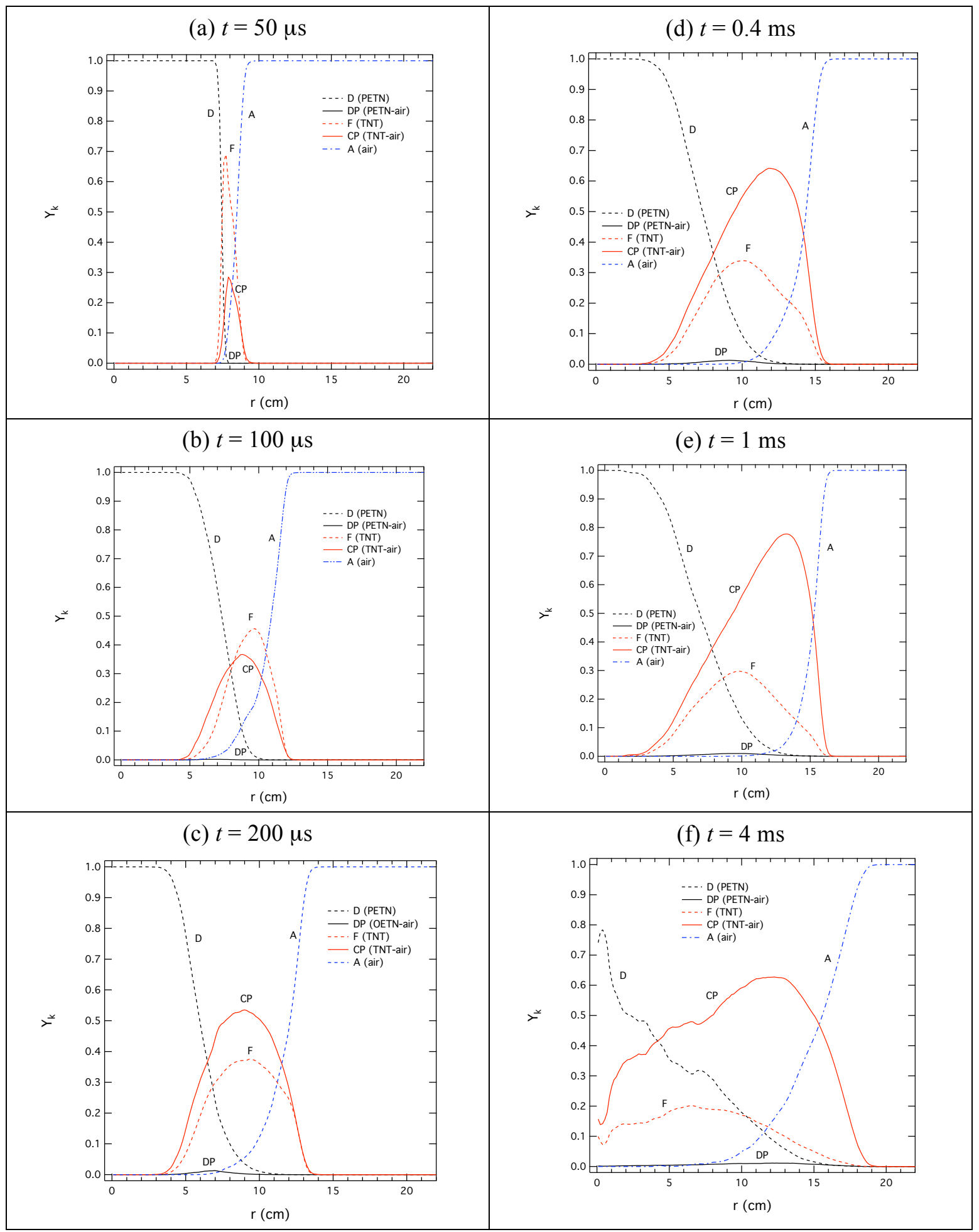

Figure 7. Evolution of the mean mass-fraction profiles $Y_{k}(r, t)$ in the combustion zone of the TNT cloud (Notation: $\mathrm{D}=$ PETN driver, $\mathrm{DP}=$ PETN-air combustion products, $\mathrm{F}=\mathrm{TNT}$ fuel, $\mathrm{CP}=\mathrm{TNT}$-air combustion products, $\mathrm{A}=$ air). 